\title{
X-Ray Microcomputed Tomography for Analysis of Retrieved Medical Devices.
}

\author{
Judd S Day ${ }^{1,2}$, Daniel W Macdonald ${ }^{2}$ and Steven M Kurtz ${ }^{1,2}$ \\ 1. Exponent, Inc., Philadelphia, PA, USA. \\ 2. Implant Research Center, Drexel University, Philadelphia, PA, USA.
}

$\mathrm{X}$-ray microcomputed tomography (microCT) is a non-destructive method that uses X-rays to reconstruct a virtual three-dimensional stack of cross-sections. The resulting image data can be used to assess material geometry, density distribution, and the presence of cracks or voids, among other properties. In this presentation, we will present case studies illustrating the use of microCT for the non-destructive analysis of retrieved medical devices.

The first case study involves investigation of suture material from a patient who had died following a carotid endarterectomy procedure. Carotid endarterectomy is a procedure that is performed to remove plaque from the carotid artery (i.e. the artery that provides blood to the brain). After removing plaque from the artery, the vessel is repaired by closing with a suture. Suture material was excised from the patient's carotid artery post mortem and analyzed using a combination of light microscopy, scanning electron microscopy and microCT to investigate the cause of failure. A "pigtail" suture end was observed by microscopy in the vicinity of the failed repair, consistent with slipping of a suboptimal surgical knot (Figure 1). Using scanning electron microscopy, it was determined that there was no evidence of failure of the suture ends due to voids or imperfections of the suture material. Further investigation of the retrieved suture material using microCT confirmed that there were no unaccounted suture ends, and that there were multiple knot throws constructed using suboptimal technique (Figure 2).

The second case study involves the use of microCT to estimate material loss (wear) and damage from retrieved polyethylene orthopaedic bearing surfaces including total hips, knees, elbows, shoulders and spinal discs. Polyethylene is frequently used as an orthopaedic bearing, but is known to wear resulting in particles that can evoke an adverse response in the surrounding tissues. Evaluation of retrieved orthopaedic devices can include quantification of polyethylene creep and damage and examination of subsurface cracks (Figure 3). Using wear data from total elbow and total disc replacements, we have been able to verify anatomical loading conditions which were then used to develop in vitro validation tests of these devices. Using microCT we have also been able to estimate the material loss from highly cross linked polyethylene knee components. Highly crosslinked polyethylene is resistant to wear in the anatomical environment and its clinical use is increasing. However, there is little clinical data quantifying the volumetric wear in vivo. We found that retrieved knee bearing surfaces may contain embedded cement debris (Figure 4). Volumetric wear could be quantified using microCT and wear maps created to characterize the locations of component wear (Figure 5). This data will be useful for further understanding of the biomechanics of clinical failure. We also used microCT to demonstrate a substantial amount of bearing surface penetration and damage was due to material creep and could be recovered by remelting the material.

We have demonstrated that microCT provides a versatile nondestructive tool for examining retrieved medical devices. 

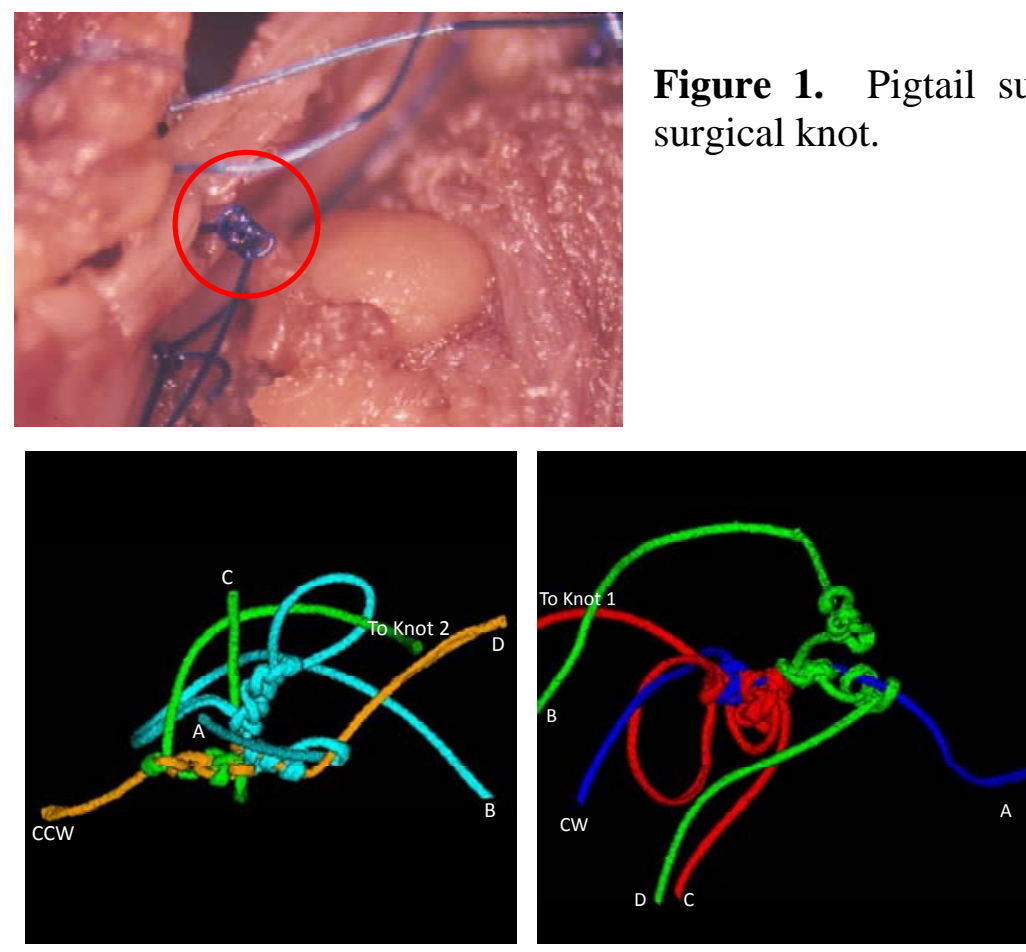

Figure 1. Pigtail suture end, consistent with slippage of the surgical knot.
Figure 2. Identification of individual knot strands from microcomputed tomography data. Once the individual knot strands were identified, it was clear that there were a number of slip knots present in the knot structure. It was also clear that knot two comprised a convoluted structure and that all suture ends were accounted for.

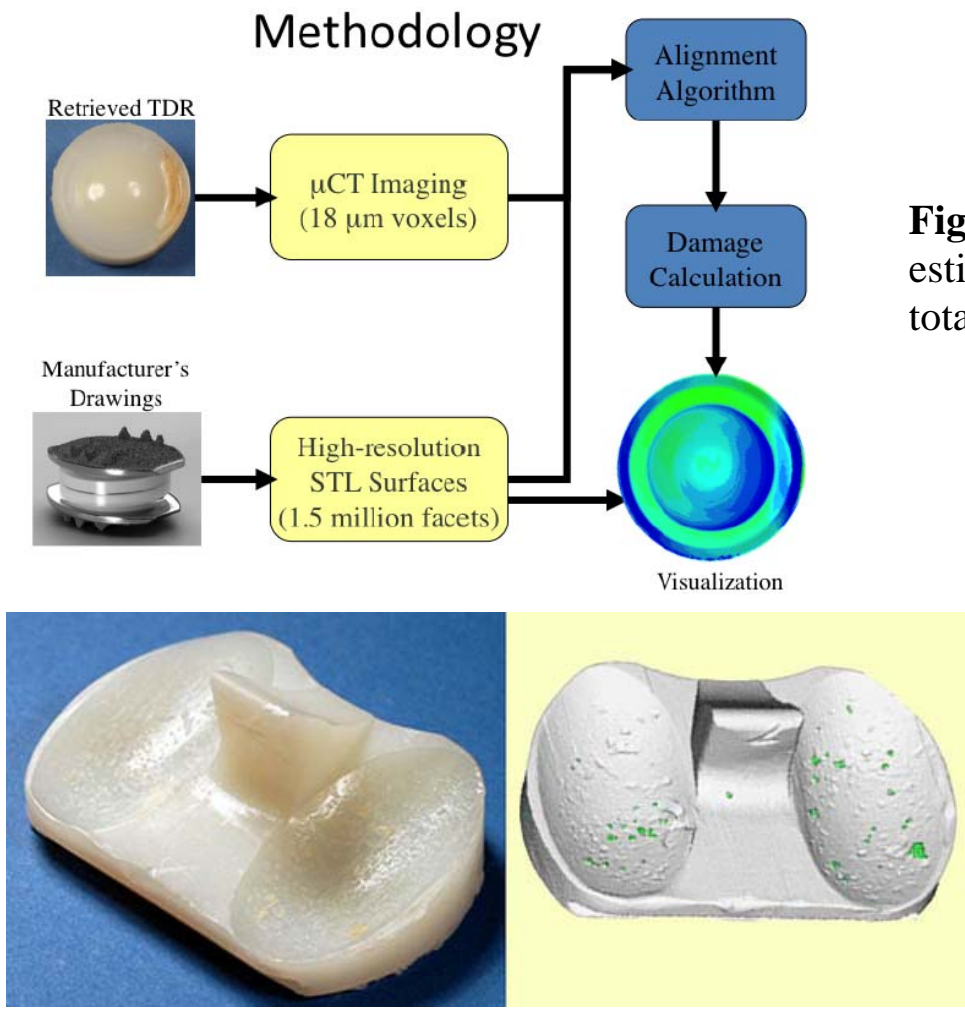

Figure 4. Total knee component with embedded third body cement debris.
Figure 3. Example of methodology for estimating the volumetric wear of a polyethylene total disc component.

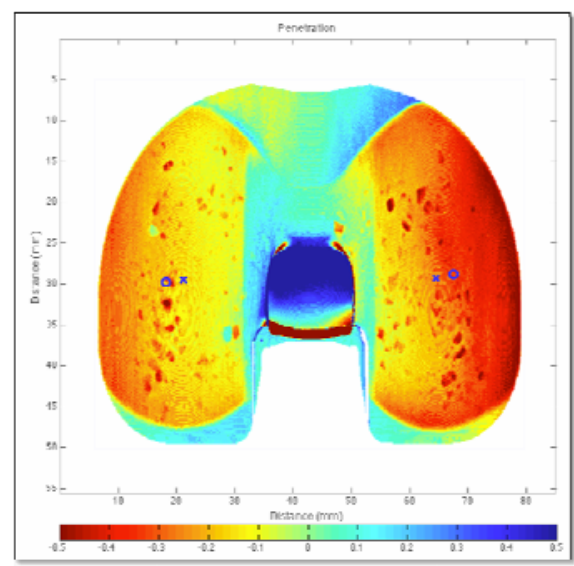

Figure 5. Penetration map for total knee component.. 